\title{
Prognostic biomarkers of malignant patients with pleural effusion: a systematic review and meta-analysis
}

Peng Peng ${ }^{1+}$, Yuan Yang ${ }^{2+}$, Juan Du ${ }^{2 \dagger}$, Kan Zhai $^{2}$ and Huan-Zhong Shi ${ }^{1,2^{*}}$

\begin{abstract}
Background: Pleural effusion is a common clinical problem in patients with cancer. We aimed to summarize all the known prognostic indicators of malignant pleural effusion.

Methods: We did a systematic review and meta-analysis with a systematic literature search. All prospective or retrospective cohort studies that estimated the prognostic factors of malignant pleural effusion were enrolled. MantelHaenszel method was used to calculate the pooled hazard ratio (HR) and 95\% confidence interval (Cl).

Results: Eventually, we identified 82 studies with a total of 10,748 patients that met our inclusion criteria. The LENT score showed a good prognostic value (HR 1.97, 95\% Cl 1.67-2.31) so did the LENT score item. In addition, clinical parameters like stage (HR 1.68, 95\% Cl 1.25-2.25), distant metastasis (HR 1.62, 95\% Cl 1.38-1.89), EGFR mutation (HR $0.65,95 \% \mathrm{Cl} 0.56-0.74$ ), serum biological parameters like hemoglobin ( $\mathrm{HR} 1.56,95 \% \mathrm{Cl} 1.17-2.06$ ), albumin (HR 1.71, 95\% Cl 1.25-2.34), C-reaction protein ( $H R$ 1.84, 95\% Cl 1.49-2.29), VEGF (HR 1.70,95\% Cl 1.18-2.43) and pleural effusion biological parameters like PH (HR 1.95, 95\% Cl 1.46-2.60), glucose (HR 1.75, 95\% Cl 1.18-2.61), VEGF (HR 1.99, 95\% Cl 1.67-2.37), and survivin ( $\mathrm{HR} 2.90,95 \% \mathrm{Cl} 1.17-7.20$ ) are also prognostic factors for malignant pleural effusion.

Conclusions: For malignant pleural effusion, LENT score and its items are valuable prognostic biomarkers, so do the clinical parameters like stage, distant metastasis, EGFR mutation, the serum biological parameters like hemoglobin, albumin, C-reaction protein, VEGF and the pleural effusion biological parameters like PH, glucose, VEGF and survivin.
\end{abstract}

Keywords: Pleural effusion, Cancer, Prognosis, Systematic review, Meta-analysis

\section{Introduction}

Pleural effusion is a common problem in many diseases especially in cancer. It occurs as a result of in situ pleural involvement and/or metastatic malignancy in the pleural cavity resulting in increased vascular permeability, production of excess fluid in excess of lymphatic reabsorption capacity, and/or disruption of lymphatic

\footnotetext{
*Correspondence: shihuanzhong@sina.com

†Peng Peng, Yuan Yang, and Juan Du contributed equally to the present work

${ }^{1}$ Department of Respiratory and Critical Care Medicine, Wuhan Pulmonary Hospital, Wuhan 430030, China

Full list of author information is available at the end of the article
}

reabsorption capacity causing fluid accumulation in the pleural cavity [1]. It accounts for greater than 125,000 hospital admissions per year in the United States and estimated inpatient costs of greater than $\$ 5$ billion per year [2]. The occurrence of pleural effusion in patients with malignancy always indicates disseminated or advanced disease [3]. In lung cancer, it upstages the severity of illness to stage IV [4] and significantly reduces life expectancy in non-small cell lung cancer [5]. The average survival of malignant pleural effusions (MPE) ranges from 4 to 7 months and is dependent on the stage and type of the underlying malignancy [6]. Increasing importance is placed on slowing down disease progression by improving risk factors. However, the factors that

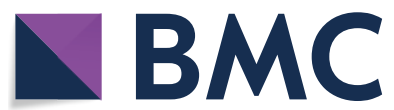

(c) The Author(s) 2022. Open Access This article is licensed under a Creative Commons Attribution 4.0 International License, which permits use, sharing, adaptation, distribution and reproduction in any medium or format, as long as you give appropriate credit to the original author(s) and the source, provide a link to the Creative Commons licence, and indicate if changes were made. The images or other third party material in this article are included in the article's Creative Commons licence, unless indicated otherwise in a credit line to the material. If material is not included in the article's Creative Commons licence and your intended use is not permitted by statutory regulation or exceeds the permitted use, you will need to obtain permission directly from the copyright holder. To view a copy of this licence, visit http://creativecommons.org/licenses/by/4.0/. The Creative Commons Public Domain Dedication waiver (http://creativeco mmons.org/publicdomain/zero/1.0/) applies to the data made available in this article, unless otherwise stated in a credit line to the data. 
define malignant progression and mortality in MPE are poorly understood.

The LENT scoring system is the first validated prognostic score in MPE. It predicts patients' survival on the basis of tumor type, pleural fluid lactate dehydrogenase (LDH), Eastern Cooperative Oncology Group Performance Status (ECOG PS), and blood neutrophil-to-lymphocyte ratio (NLR) and predicts survival with significantly better accuracy than ECOG PS alone [7]. Another prognostic model for MPE is the PROMISE score which combines biological and clinical parameters to accurately estimate 3-month mortality [8]. The modified LENT score was based on LENT score replacing the "tumor type" score of 2 with 0 in patients with lung adenocarcinoma to illustrated that the actual survival in patients having MPE from lung adenocarcinoma was higher than predicted by the LENT score [9]. A new prognostic model-SELECT prognostication model was proposed recently with high accuracy at identifying patients with high probability of survival at 90 days an Asian population [10]. Notably, EGFR mutations were included in prediction model for the first time. These findings are consistent with our meta-analysis that EGFR is a protective factor for lung cancer. In addition, minimal pleural effusion itself is also an important prognostic factor of worse survival, especially in early-stage malignant disease [11]. Our group has systematically studied the prognostic role of pleural effusion in malignancy and found that whether malignant effusion is clearly diagnosed with cytological or histological examination, pleural effusion is a prognostic factor associated with a poor prognosis for cancer patients. Thus, capturing clinical parameter biomarkers, plasma biomarkers and pleural effusion biomarkers are increasingly important.

We aim to systematically synthesize the published evidence on the associations between the prognostic biomarkers and clinical outcomes in patients with malignant pleural effusion to provide a new insight for development of scoring systems. To our knowledge, no published study has thoroughly and systematically summarized these evidences.

\section{Methods}

\section{Search strategy and selection criteria}

We conducted a systematic review and meta-analysis to assess the associations between the prognostic factors and clinical outcomes in malignant patients with pleural effusion. The search flow diagram for this meta-analysis is shown in Fig. 1. Databases searched included PubMed, Cochrane Library, Medline (accessed via OVID), Embase, and Web of Science, covering all dates from the creation of each database up to April 2, 2020. The index terms included "pleural effusion", "malignant", and "prognosis", as well as the related words. Additional file 1: eTable 1 presents the detailed search strategy. Additional studies were identified by searching the list of references of included studies, as well as previous relevant metaanalysis and systematic reviews. This meta-analysis was carried out following Preferred Reporting Items for Systematic Reviews and Meta-Analysis (PRISMA) guidelines [12].

\section{Eligibility criteria}

We enrolled prospective or retrospective cohort studies in English that estimated the association between prognostic factors and clinical outcomes in malignant patients with pleural effusion. There were no restrictions on studies with respect to settings, tumor types, or comorbidity types. Inclusion criteria were as follows: (1) study population: patients diagnosed with any type of malignancy and pleural effusion; (2) target: assessing a relevant biomarker; (3) outcomes: overall survival (OS), progression-free survival (PFS); (4) study type: prospective or retrospective cohort studies. The exclusion criteria included (1) studies involving infants, children and adolescents (2) no clearly reported diagnostic criteria for malignant tumor or pleural effusion; (3) narrative reviews, comments, editorials, case reports, meeting abstracts, guidelines or corresponding letters; and (4) full-text paper unavailable in English; (5) sample size $<20$. The methods were defined in advance in the original study protocol (Additional file 1, pp 1-2).

\section{Data extraction and quality assessment}

Two authors ( $Y Y$ and DJ) independently screened the title and abstract of the literature retrieved from the databases by the search strategy. Then YY and DJ independently reviewed the full text of articles and assessed articles for eligibility according to the inclusion criteria. Disagreements between the two authors were settled by of arbitration of the principal investigator. Two authors extracted data from included studies using a standardized form based on the Cochrane Consumers and Communication Review Group's data extraction template. The data extracted by the two authors was cross-checked and differences were resolved by checking the original article. Where data were not enough to extract, the corresponding authors were contacted and asked to provide data.

Data extracted included: (1) clinical characteristics (including age, gender, country, publication year, sample size, and primary tumor type); (2) all kinds of prognostic factors; (3) clinical outcomes (OS and PFS). Cox proportional hazards modeling results of hazard ratio (HR) and 95\% confidence interval (CI) of prognostic factors were extracted and we applied the software Origin (version 2020; https:/www.originlab.com/) to digitize and extract 


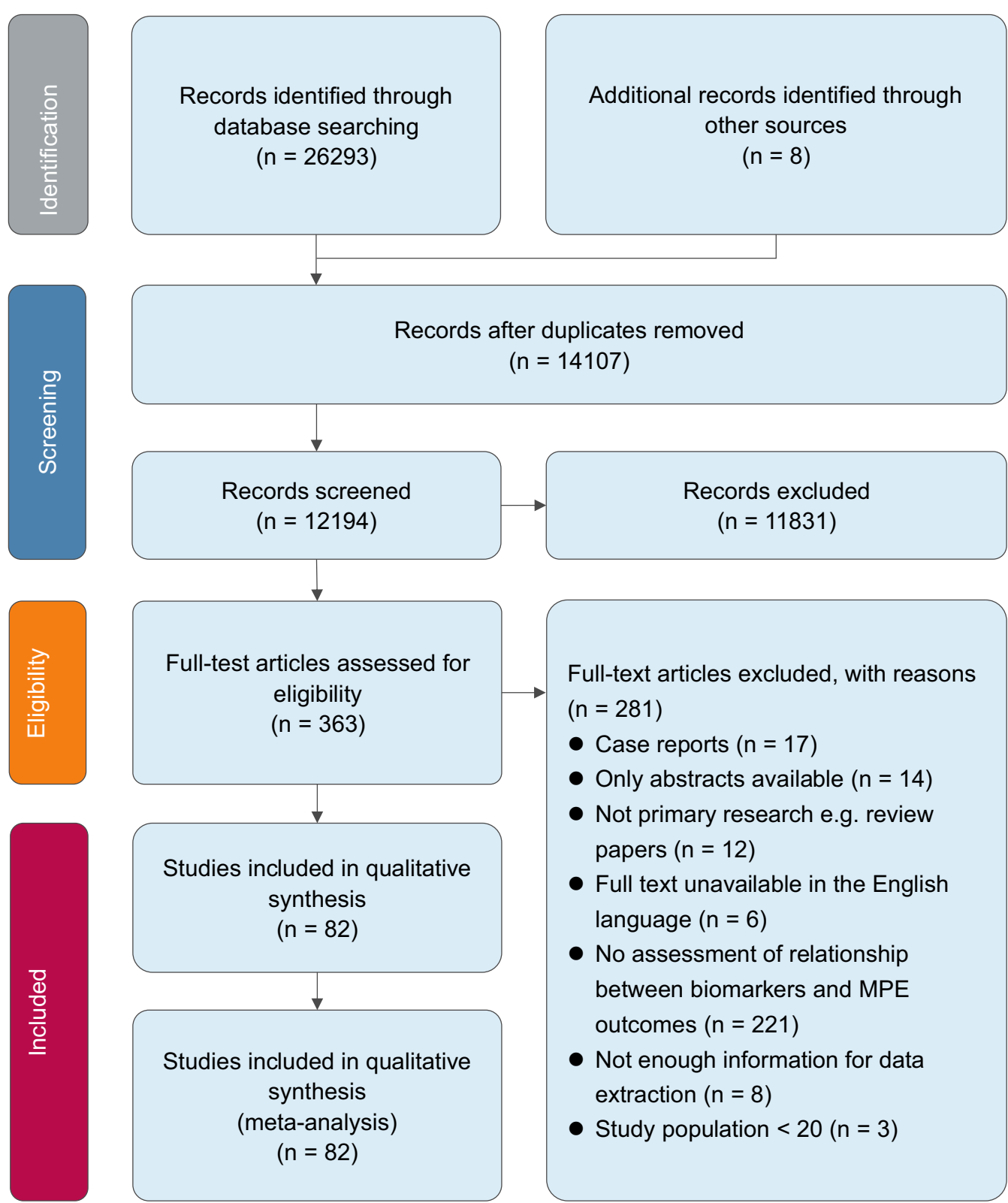

Fig. 1 The PRISMA diagram for study selection. PRISMA: Preferred Reporting Items for Systematic Reviews and Meta-Analyses

key data from the published Kaplan-Meier curves. When HR, 95\% CI and Kaplan-Meier curves were not given directly, the data extract method was based on the method of Parmar et al. [13].

The quality of each study was assessed in accordance with the Newcastle-Ottawa Scale (NOS) [14]. As all included studies are cohort studies, the scoring was based on the following items: (1) selection: representativeness of exposed cohort, selection of non-exposed cohort, ascertainment of exposure, demonstration that outcome of interest was not present at start of study; (2) comparability: comparability of cohorts on the basis of the design or analysis; (3) outcome: assessment of outcome, was follow-up long enough for outcomes to occur and adequacy of follow up of cohorts. Two reviews (YY and DJ) independently assessed the risk of bias of each trial. They cross checked the data and settled discrepancies by discussion.

\section{Statistical analysis}

The pooled HR and 95\% CIs were calculated using Mantel-Haenszel method. A random-effects model was used 
when significant heterogeneity was observed $\left(\mathrm{I}^{2}>50 \%\right)$; otherwise we used a fixed-effects mode. We further performed sensitivity analysis by omitting one study at a time and examining the influence of each study on the pooled estimates of the primary outcome. In addition, we generated contour-enhanced meta-analysis funnel plots to assess potential publication bias or other biases associated with trial size. A two-sided $\mathrm{P}$ value $<0.05$ was considered statistically significant. The data analyses were performed using software Stata (version 15; https://www. stata.com/).

\section{Results}

\section{Study selection and characteristics}

The systematic review yielded 14,107 references from five electronic databases. Eventually, we identified 82 studies with a total of 10,748 patients that met our inclusion criteria. The sample size ranged from 23 to 789 subjects. The following period ranged from 1 to 264 months. The numbers of studies comparing the OS and PFS difference between different demographic data in malignant patients with pleural effusion were 78 and 9, respectively. All 83 studies included in this systematic review were cohort studies. Figure 1 presents the PRISMA diagram of study selection. All included studies were listed in Additional file 1 (pp 3-8) and their basic characteristics were listed in Table 1.

\section{Quality assessment of individual studies}

The quality of each study was assessed in accordance with the Newcastle-Ottawa Scale (NOS) and was summarized in Additional file 1: eTable 2. The NOS score of all involved studies were above 6 , which indicate low risk of bias.

\section{Included biomarkers}

To comprehensively analyze the prognostic factors of malignant patients with pleural effusion, we included all biomarkers with the number of studies more than three. The clinical parameter biomarkers of OS include age, gender, smoking status, ECOG PS, stage, histology, cytology, distant metastasis, EGFR mutation and LENT score. The clinical parameter biomarkers of PFS include age, gender, smoking status, ECOG PS, stage and EGFR mutation. In addition, many studies analyzed the serum and pleural effusion biomarkers in the prognostic value of malignant patients with pleural effusion. The serum biomarkers include white blood cell counts (WBC), NLR, hemoglobin, total protein, albumin, LDH, C-reactive protein (CRP) and vascular endothelial growth factor (VEGF). The pleural effusion biomarkers include neutrophils, $\mathrm{PH}$, total protein, albumin, glucose, LDH, VEGF and survivin.

\section{Clinical parameter biomarkers for malignant patients} with pleural effusion, primary outcome: OS

In studies that reported the OS as outcome and evaluated the prognostic value of clinical parameter biomarkers, 36 were about the prognostic value of age, 35 of gender, 18 of smoking status, 28 of ECOG PS, 14 of stage, 17 of histology, 7 of cytology, 11 of distant metastasis, 7 of EGFR mutation and 8 of LENT score.

Figure 2a shows the forest plot of pooled HRs for the prognostic value of demographic data in OS with 95\% CI. The pooled data demonstrate that elder age (HR 1.07; 95\% CI 1.02-1.12), male gender (HR 1.11; 95\% CI 1.05-1.17), smokers (HR 1.18; 95\% CI 1.04-1.33), high ECOG PS (HR 2.35; 95\% CI 1.83-3.00), M1b stage (HR 1.68; 95\% CI 1.25-2.25), non-adenocarcinoma (HR 1.46; 95\% CI 1.20-1.78), positive distant metastasis (HR 1.62; 95\% CI 1.38-1.89) and high LENT score (HR 1.97; 95\% CI 1.67-2.31) are prognostic risk factor in OS for malignant patients with pleural effusion. On the other hand, positive EGFR mutation is a protective factor in OS for malignant patients with pleural effusion (HR 0.65; 95\% CI 0.56-0.74). The forest plots of each biomarker are showed on Additional file 1: eFigure 1-10. Heterogeneity testing revealed heterogeneity $\left(I^{2}>50 \%\right)$ in age, smoking status, ECOG PS, stage and histology.

\section{Clinical parameter biomarkers for malignant patients with pleural effusion, secondary outcome: PFS.}

In studies that reported the PFS as outcome and evaluated the prognostic value of clinical parameters, 4 were about the prognostic value of age, 4 of gender, 5 of smoking status, 4 of ECOG PS, 4 of stage and 5 of EGFR mutation.

Figure $2 \mathrm{~b}$ shows the forest plot of pooled HRs for the prognostic value of demographic data in PFS with $95 \%$ CI. The pooled data demonstrate that elder age (HR 1.35; 95\% CI 1.04-1.74), smokers (HR 1.15; 95\% CI 1.00-1.31) and M1b stage (HR 1.89; 95\% CI 1.01-3.52) are prognostic risk factors in PFS for malignant patients with pleural effusion. Positive EGFR mutation is still a protective factor in PFS for lung adenocarcinoma patients with pleural effusion (HR 0.69; 95\% CI 0.54-0.89). The forest plots of each biomarker are showed on Additional file 1: eFigure 11-16. Heterogeneity testing revealed heterogeneity $\left(I^{2}>50 \%\right)$ in ECOG PS and stage.

\section{Serum prognostic biomarkers for malignant patients with pleural effusion}

Serum prognostic biomarkers has been widely studied in the few decades and we systematically summarized these studies. In these studies, 4 were about the prognostic value of WBC, 5 of NLR, 4 of hemoglobin, 3 of total protein, 7 of albumin, 6 of LDH, 4 of CRP and 4 of VEGF. 
Table 1 Baseline Characteristics of the Included Studies

\begin{tabular}{|c|c|c|c|c|c|c|c|c|}
\hline Study & Year & Country & Age & Sex & Sample size & $\begin{array}{l}\text { Follow-up } \\
\text { (months) }\end{array}$ & Cancer type & Outcome \\
\hline Sahn et al & 1988 & USA & $60.0 \pm 1.9$ & $\begin{array}{l}\text { Mixed (53.33\% } \\
\text { male) }\end{array}$ & 60 & 37 & $\begin{array}{l}\text { Mixed (not speci- } \\
\text { fied) }\end{array}$ & OS \\
\hline Panadero et al & 1989 & Spain & NA & NA & 50 & 28 & $\begin{array}{l}\text { Pleural metastatic } \\
\text { carcinoma }\end{array}$ & OS \\
\hline Foresti et al & 1990 & Italy & $64.8 \pm 17.7$ & $\begin{array}{l}\text { Mixed ( } 44.44 \% \\
\text { male) }\end{array}$ & 36 & 27 & $\begin{array}{l}\text { Mixed (lung, } \\
\text { breast, mesothe- } \\
\text { lioma, and others) }\end{array}$ & OS \\
\hline Gottehrer et al & 1991 & USA & NA & NA & 26 & 7 & MPM & OS \\
\hline Sugiura et al & 1997 & Japan & NA & $\begin{array}{l}\text { Mixed }(67.51 \% \\
\text { male) }\end{array}$ & 62 & 54 & NSCLC & OS \\
\hline Moragón et al & 1998 & Spain & $60 \pm 13$ & Mixed (45\% male) & 120 & median 9 & $\begin{array}{l}\text { Mixed (NSCLC, } \\
\text { breast, lymphoma, } \\
\text { and others) }\end{array}$ & OS \\
\hline Burrows et al & 2000 & USA & $62(24-84)$ & Mixed (50\% male) & 85 & 53 & $\begin{array}{l}\text { Mixed (lung, } \\
\text { breast, mesothe- } \\
\text { lioma, and others) }\end{array}$ & OS \\
\hline Heffner et al & 2000 & USA & $61 \pm 13$ & Mixed (50\% male) & 417 & 36 & $\begin{array}{l}\text { Mixed (lung, } \\
\text { breast, unknown } \\
\text { primary, mesothe- } \\
\text { lioma, and others) }\end{array}$ & OS \\
\hline Chen et al & 2001 & China & $67.9 \pm 11.2$ & $\begin{array}{l}\text { Mixed }(71.29 \% \\
\text { male) }\end{array}$ & 202 & 49 & Lung cancer & OS \\
\hline Thyle'n et al & 2001 & Sweden & NA & Mixed (97\% male) & 100 & 100 & MPM & OS \\
\hline Bernard et al & 2002 & France & $65 \pm 11$ & $\begin{array}{l}\text { Mixed }(45.71 \% \\
\text { male) }\end{array}$ & 70 & $>3$ & $\begin{array}{l}\text { Mixed (breast, } \\
\text { unknown, lung, } \\
\text { and others) }\end{array}$ & OS \\
\hline Eitan et al & 2005 & USA & $55(26-88)$ & NA & 97 & 150 & $\begin{array}{l}\text { Optimally } \\
\text { debulked ovarian } \\
\text { carcinoma }\end{array}$ & PFS \\
\hline Aelony et al & 2006 & USA & NA & $\begin{array}{l}\text { Mixed }(92.31 \% \\
\text { male) }\end{array}$ & 26 & 18 & MPM & OS \\
\hline Aoe et al & 2006 & Japan & 69(22-95) & $\begin{array}{l}\text { Mixed }(72.55 \% \\
\text { male) }\end{array}$ & 102 & 22 & Lung cancer & OS \\
\hline Soh et al & 2006 & Japan & NA & $\begin{array}{l}\text { Mixed }(65.6 \% \\
\text { male) }\end{array}$ & 61 & 30 & Lung cancer & OS \\
\hline Bielsa et al & 2008 & Spain & $67 \pm 13$ & $\begin{array}{l}\text { Mixed }(52.82 \% \\
\text { male) }\end{array}$ & 284 & 40 & $\begin{array}{l}\text { Mixed (lung, } \\
\text { breast, unknown, } \\
\text { and others) }\end{array}$ & OS \\
\hline Wu et al & 2008 & China & $63.4(37.5-85.4)$ & $\begin{array}{l}\text { Mixed }(38.97 \% \\
\text { male) }\end{array}$ & 136 & 27 & $\begin{array}{l}\text { Lung adenocarci- } \\
\text { noma }\end{array}$ & OS \\
\hline Hsu et al & 2009 & China & $63(27-80)$ & $\begin{array}{l}\text { Mixed (51.5\% } \\
\text { male) }\end{array}$ & 97 & 49 & NSCLC & OS \\
\hline Wu et al & 2009 & China & NA & $\begin{array}{l}\text { Mixed (58.33\% } \\
\text { male) }\end{array}$ & 60 & 36 & Lung cancer & OS \\
\hline Kotyza et al & 2010 & Czech Republic & $63 \pm 11$ & $\begin{array}{l}\text { Mixed }(70.73 \% \\
\text { male) }\end{array}$ & 164 & 36 & Lung cancer & OS \\
\hline Lan et al & 2010 & China & $59 \pm 16$ & Mixed (55\% male) & 44 & 36 & $\begin{array}{l}\text { Mixed (lung, } \\
\text { breast, hepatoma } \\
\text { and others) }\end{array}$ & OS \\
\hline Ozyurtkan et al & 2010 & Turkey & $59 \pm 14$ & Mixed (56\% male) & 85 & 52 & $\begin{array}{l}\text { Mixed (mesothe- } \\
\text { lioma, lung, ovary, } \\
\text { breast, and others) }\end{array}$ & OS \\
\hline Pilling et al & 2010 & UK & $60(26-89)$ & $\begin{array}{l}\text { Mixed (38.85\% } \\
\text { male) }\end{array}$ & 278 & 71 & $\begin{array}{l}\text { Mixed (breast, } \\
\text { mesothelioma, } \\
\text { lung, ovarian, and } \\
\text { others) }\end{array}$ & OS \\
\hline
\end{tabular}


Table 1 (continued)

\begin{tabular}{|c|c|c|c|c|c|c|c|c|}
\hline Study & Year & Country & Age & Sex & Sample size & $\begin{array}{l}\text { Follow-up } \\
\text { (months) }\end{array}$ & Cancer type & Outcome \\
\hline Tanrikulu et al & 2010 & Turkey & NA & $\begin{array}{l}\text { Mixed (59.8\% } \\
\text { male) }\end{array}$ & 363 & 54 & MPM & OS \\
\hline Hirayama et al & 2010 & Japan & $69.17 \pm 9.64$ & $\begin{array}{l}\text { Mixed ( } 82.6 \% \\
\text { male) }\end{array}$ & 54 & 20 & MPM & OS \\
\hline Park et al & 2011 & South Korea & $68.3 \pm 15.0$ & $\begin{array}{l}\text { Mixed }(65.67 \% \\
\text { male) }\end{array}$ & 67 & 36 & Lung cancer & OS \\
\hline Sakr et al & 2011 & France & Median 61 & $\begin{array}{l}\text { Mixed }(46.7 \% \\
\text { male) }\end{array}$ & 107 & 120 & $\begin{array}{l}\text { Mixed (lung, } \\
\text { melanoma, breast, } \\
\text { ovarian and } \\
\text { others) }\end{array}$ & OS \\
\hline Yamada et al & 2011 & Japan & $66.16 \pm 10.05$ & $\begin{array}{l}\text { Mixed }(68.9 \% \\
\text { male) }\end{array}$ & 45 & 73 & MPM & OS \\
\hline Guo et al & 2011 & China & $64.5 \pm 9.8$ & $\begin{array}{l}\text { Mixed (53.13\% } \\
\text { male) }\end{array}$ & 128 & 28 & $\begin{array}{l}\text { Lung adenocarci- } \\
\text { noma }\end{array}$ & OS \\
\hline Hooper et al & 2012 & UK & 73(39-96) & $\begin{array}{l}\text { Mixed }(62.14 \% \\
\text { male) }\end{array}$ & 103 & 6 & $\begin{array}{l}\text { Mixed (mesothe- } \\
\text { lioma, lung, breast, } \\
\text { ovarian, and } \\
\text { others) }\end{array}$ & OS \\
\hline Maribel et al & 2012 & spain & 63(53.2-80.0) & $\begin{array}{l}\text { Mixed }(66.7 \% \\
\text { male) }\end{array}$ & 30 & 40 & $\begin{array}{l}\text { Lung adenocarci- } \\
\text { noma }\end{array}$ & OS \\
\hline Qian et al & 2012 & China & NA & $\begin{array}{l}\text { Mixed }(60.76 \% \\
\text { male) }\end{array}$ & 79 & 18 & $\begin{array}{l}\text { Lung adenocarci- } \\
\text { noma }\end{array}$ & OS \\
\hline Qian et al & 2012 & China & NA & Mixed (61\% male) & 103 & 7 & $\begin{array}{l}\text { Lung adenocarci- } \\
\text { noma }\end{array}$ & PFS \\
\hline Wang et al & 2012 & China & NA & $\begin{array}{l}\text { Mixed ( } 41.85 \% \\
\text { male) }\end{array}$ & 184 & 29 & NSCLC & OS \\
\hline Cheng et al & 2013 & China & NA & $\begin{array}{l}\text { Mixed (63.38\% } \\
\text { male) }\end{array}$ & 71 & 60 & Lung cancer & OS \\
\hline Faiz et al & 2013 & USA & $65(17-85)$ & $\begin{array}{l}\text { Mixed }(54.95 \% \\
\text { male) }\end{array}$ & 111 & 172 & Acute leukemia & OS \\
\hline Gorgun et al & 2013 & Turkey & $60.20 \pm 13.91$ & Mixed (57\% male) & 51 & 27 & $\begin{array}{l}\text { Mixed (lung, } \\
\text { breast, pancreas, } \\
\text { and others) }\end{array}$ & OS \\
\hline Park et al & 2013 & Korea & $68.3 \pm 15.0$ & $\begin{array}{l}\text { Mixed (66.25\% } \\
\text { male) }\end{array}$ & 80 & 35 & Lung cancer & OS \\
\hline Wu et al & 2013 & China & 27.9-95.5 & $\begin{array}{l}\text { Mixed }(45.5 \% \\
\text { male) }\end{array}$ & 448 & 81 & $\begin{array}{l}\text { Lung adenocarci- } \\
\text { noma }\end{array}$ & OS \\
\hline Anevlavis et al & 2014 & Greece & $69(37-93)$ & Mixed (53\% male) & 90 & 56 & $\begin{array}{l}\text { Mixed (breast, } \\
\text { mesothelioma, } \\
\text { gastrointestinal, } \\
\text { and others) }\end{array}$ & OS \\
\hline Clive et al & 2014 & UK & $53-80$ & $\begin{array}{l}\text { Mixed (53.6\% } \\
\text { male) }\end{array}$ & 789 & 33 & $\begin{array}{l}\text { Mixed (mesotheli- } \\
\text { oma, hematologi- } \\
\text { cal malignancy, } \\
\text { gynecological } \\
\text { malignancy, } \\
\text { breast, and others) }\end{array}$ & OS \\
\hline Ni et al & 2014 & China & $31-81$ & Mixed (47\% male) & 75 & 45 & NSCLC & OS \\
\hline Xu et al & 2014 & China & $56.3 \pm 12.5$ & $\begin{array}{l}\text { Mixed }(46.15 \% \\
\text { male) }\end{array}$ & 78 & 16 & Lung cancer & OS \\
\hline Zhang et al & 2014 & China & $64(36-84)$ & Mixed (51\% male) & 85 & 30 & NSCLC & OS \\
\hline Zhang et al & 2014 & China & Median 64 & $\begin{array}{l}\text { Mixed }(65.7 \% \\
\text { male) }\end{array}$ & 70 & 36 & Lung cancer & OS \\
\hline Abrao et al & 2015 & Brazil & $59.6(11.8)$ & $\begin{array}{l}\text { Mixed }(29.07 \% \\
\text { male) }\end{array}$ & 86 & 1 & $\begin{array}{l}\text { Mixed (lung, } \\
\text { breast, gastro- } \\
\text { intestinal, and } \\
\text { others) }\end{array}$ & OS \\
\hline
\end{tabular}


Table 1 (continued)

\begin{tabular}{|c|c|c|c|c|c|c|c|c|}
\hline Study & Year & Country & Age & Sex & Sample size & $\begin{array}{l}\text { Follow-up } \\
\text { (months) }\end{array}$ & Cancer type & Outcome \\
\hline \multirow[t]{2}{*}{ Gkiozos et al } & \multirow[t]{2}{*}{2015} & \multirow[t]{2}{*}{ Greece } & \multirow[t]{2}{*}{ NA } & \multirow{2}{*}{$\begin{array}{l}\text { Mixed }(75.2 \% \\
\text { male) }\end{array}$} & \multirow[t]{2}{*}{40} & \multirow[t]{2}{*}{44} & \multirow[t]{2}{*}{ NSCLC } & OS \\
\hline & & & & & & & & PFS \\
\hline Porcel et al & 2015 & Spain & $58-78$ & Mixed (77\% male) & 556 & 30 & Lung cancer & OS \\
\hline Xu et al & 2015 & China & $58.3 \pm 13.7$ & $\begin{array}{l}\text { Mixed }(54.08 \% \\
\text { male) }\end{array}$ & 98 & 100 & Lung cancer & OS \\
\hline Zamboni et al & 2015 & Brazil & $60.0(1.0-95.0)$ & Mixed (47\% male) & 165 & 100 & $\begin{array}{l}\text { Mixed (ovary, } \\
\text { breast, lymphoma, } \\
\text { lung, and others) }\end{array}$ & OS \\
\hline Zhao et al & 2015 & China & NA & $\begin{array}{l}\text { Mixed ( } 48.8 \% \\
\text { male) }\end{array}$ & 43 & 30 & $\begin{array}{l}\text { Lung adenocarci- } \\
\text { noma }\end{array}$ & PFS \\
\hline Abrao et al & 2016 & Brazil & $60(24-86)$ & $\begin{array}{l}\text { Mixed }(31.25 \% \\
\text { male) }\end{array}$ & 64 & 6 & $\begin{array}{l}\text { Mixed (lung, } \\
\text { breast, gastro- } \\
\text { intestinal, and } \\
\text { others) }\end{array}$ & OS \\
\hline Hsu et al & 2016 & China & Median 57 & Mixed (59\% male) & 61 & 59 & $\begin{array}{l}\text { Mixed (lung, } \\
\text { breast, and others) }\end{array}$ & OS \\
\hline Kasapoglu et al & 2016 & Turkey & $64(30-85)$ & Mixed (76\% male) & 199 & 60 & Lung cancer & OS \\
\hline Psallidas et al & 2016 & UK & NA & NA & 75 & 28 & $\begin{array}{l}\text { Mixed (not speci- } \\
\text { fied) }\end{array}$ & OS \\
\hline Tamiya et al & 2016 & Greece & $68(49-83)$ & $\begin{array}{l}\text { Mixed (69.9\% } \\
\text { male) }\end{array}$ & 23 & 47 & NSCLC & $\begin{array}{l}\text { OS } \\
\text { PFS }\end{array}$ \\
\hline Terra et al & 2016 & USA & $58.9 \pm 12$ & $\begin{array}{l}\text { Mixed }(28.21 \% \\
\text { male) }\end{array}$ & 156 & 40 & $\begin{array}{l}\text { Mixed (breast, } \\
\text { lung, lymphoma, } \\
\text { and others) }\end{array}$ & OS \\
\hline \multirow[t]{2}{*}{ Usui et al } & \multirow[t]{2}{*}{2016} & \multirow[t]{2}{*}{ Japan } & \multirow[t]{2}{*}{ NA } & \multirow[t]{2}{*}{ Mixed (80\% male) } & \multirow[t]{2}{*}{25} & \multirow[t]{2}{*}{50} & \multirow[t]{2}{*}{ NSCLC } & OS \\
\hline & & & & & & & & PFS \\
\hline Verma et al & 2016 & Singapore & $71(38-92)$ & Mixed (51\% male) & 71 & 49 & $\begin{array}{l}\text { Lung adenocarci- } \\
\text { noma }\end{array}$ & OS \\
\hline Yang et al & 2016 & China & $38-75$ & $\begin{array}{l}\text { Mixed ( } 48.7 \% \\
\text { male) }\end{array}$ & 78 & 32 & Lung cancer & OS \\
\hline Amn et al & 2017 & Indonesia & $17-85$ & Mixed (44\% male) & 102 & 27 & $\begin{array}{l}\text { Mixed (lung, } \\
\text { breast, lymphoma, } \\
\text { and others) }\end{array}$ & OS \\
\hline Lee et al & 2017 & South Korea & NA & $\begin{array}{l}\text { Mixed (51.3\% } \\
\text { male) }\end{array}$ & 158 & 72 & Lung cancer & OS \\
\hline Lu et al & 2017 & China & $59.34 \pm 1.56$ & $\begin{array}{l}\text { Mixed }(65.71 \% \\
\text { male) }\end{array}$ & 70 & 264 & NSCLC & OS \\
\hline Yang et al & 2017 & Korea & $71(42-94)$ & $\begin{array}{l}\text { Mixed (37.5\% } \\
\text { male) }\end{array}$ & 40 & 40 & $\begin{array}{l}\text { Lung adenocarci- } \\
\text { noma }\end{array}$ & PFS \\
\hline Zheng et al & 2017 & China & NA & $\begin{array}{l}\text { Mixed ( } 46.1 \% \\
\text { male) }\end{array}$ & 128 & 55 & NSCLC & $\begin{array}{l}\text { OS } \\
\text { PFS }\end{array}$ \\
\hline $\begin{array}{l}\text { Abisheganaden } \\
\text { et al }\end{array}$ & 2018 & Singapore & $72(38-92)$ & Mixed (53\% male) & 70 & 20 & $\begin{array}{l}\text { Lung adenocarci- } \\
\text { noma }\end{array}$ & OS \\
\hline Elena et al & 2018 & Spain & $61.6 \pm 11.2$ & Mixed (56\% male) & 84 & 14 & $\begin{array}{l}\text { Mixed (breast, } \\
\text { mesothelioma, } \\
\text { and lung cancer) }\end{array}$ & OS \\
\hline Han et al & 2018 & Korea & $70 \pm 11$ & Mixed (65\% male) & 131 & 84 & $\begin{array}{l}\text { Mixed (lung, } \\
\text { breast, ovary, } \\
\text { lymphoma, and } \\
\text { others) }\end{array}$ & OS \\
\hline Jeba et al & 2018 & India & median 53 & Mixed (29\% male) & 48 & 70 & $\begin{array}{l}\text { Mixed (lung, } \\
\text { breast, gastro- } \\
\text { intestinal, and } \\
\text { others) }\end{array}$ & OS \\
\hline
\end{tabular}


Table 1 (continued)

\begin{tabular}{|c|c|c|c|c|c|c|c|c|}
\hline Study & Year & Country & Age & Sex & Sample size & $\begin{array}{l}\text { Follow-up } \\
\text { (months) }\end{array}$ & Cancer type & Outcome \\
\hline Lim et al & 2018 & Korea & $68(35-92)$ & $\begin{array}{l}\text { Mixed (55.3\% } \\
\text { male) }\end{array}$ & 217 & 32 & NSCLC & $\begin{array}{l}\text { OS } \\
\text { PFS }\end{array}$ \\
\hline Porcel et al & 2018 & Spain & $52-76$ & Mixed (57\% male) & 24 & 70 & $\begin{array}{l}\text { diffuse large B-cell } \\
\text { lymphomas }\end{array}$ & OS \\
\hline Psallidas et al & 2018 & UK & NA & NA & 232 & 133 & MPE & OS \\
\hline Wu et al & 2018 & China & $44(28-50)$ & Mixed (51\% male) & 142 & 45 & $\begin{array}{l}\text { Lung adenocarci- } \\
\text { noma }\end{array}$ & OS \\
\hline Xu et al & 2018 & China & $62(40-78)$ & Mixed (50\% male) & 40 & 43 & MPM & OS \\
\hline Foote et al & 2019 & USA & $61.44 \pm 15.36$ & Mixed ( $41 \%$ male) & 686 & 83 & $\begin{array}{l}\text { Mixed (lung, } \\
\text { breast, gyneco- } \\
\text { logic, lymphoma, } \\
\text { and others) }\end{array}$ & OS \\
\hline Kleontas et al & 2019 & United Kingdom & $61.0 \pm 10.9$ & $\begin{array}{l}\text { Mixed }(92.5 \% \\
\text { male) }\end{array}$ & 40 & 41 & Lung cancer & OS \\
\hline Tian et al & 2019 & China & $59.7 \pm 9.12$ & Mixed ( $46 \%$ male) & 160 & 25 & $\begin{array}{l}\text { Mixed (lung, } \\
\text { breast, esopha- } \\
\text { geal, gastric, and } \\
\text { mesothelioma) }\end{array}$ & OS \\
\hline Wang et al & 2019 & China & NA & Mixed ( $88 \%$ male) & 295 & 84 & $\begin{array}{l}\text { Lung adenocarci- } \\
\text { noma }\end{array}$ & OS \\
\hline Martin et al & 2020 & UK & $71(69-74)$ & Mixed (67\% male) & 97 & 75 & $\begin{array}{l}\text { Mixed (mesothe- } \\
\text { lioma, lung, breast, } \\
\text { genitourinary, and } \\
\text { others) }\end{array}$ & OS \\
\hline Quek et al & 2020 & Singapore & $65(56-71)$ & Mixed (59\% male) & 130 & 14 & $\begin{array}{l}\text { Mixed (lung, mes- } \\
\text { othelioma, breast, } \\
\text { gastrointestinal, } \\
\text { and others) }\end{array}$ & OS \\
\hline Shi et al & 2020 & China & $65(55-73)$ & $\begin{array}{l}\text { Mixed (51.3\% } \\
\text { male) }\end{array}$ & 193 & 65 & $\begin{array}{l}\text { Mixed (lung, } \\
\text { mesothelioma, } \\
\text { and others) }\end{array}$ & OS \\
\hline Stockhammer et al & 2020 & Germany & $73.7 \pm 8.6$ & Mixed ( $88 \%$ male) & 48 & 40 & MPM & OS \\
\hline
\end{tabular}

NA not available, NSCLC non-small cell lung cancer, MPM malignant pleural mesothelioma, OS overall survival, PFS progression-free survival

The pooled data of forest plot is showed in Fig. 3. The results demonstrate that high NLR (HR 2.17; 95\% CI 1.22-3.88), low hemoglobin (HR 1.56; 95\% CI 1.172.06), low total protein (HR 1.14; 95\% CI 1.07-1.23), low albumin (HR 1.71; 95\% CI 1.25-2.34), high LDH (HR 1.54; 95\% CI 1.08-2.19), high CRP (HR 1.84; 95\% CI 1.49-2.29) and high VEGF (HR 1.70; 95\% CI 1.182.43) in serum are prognostic risk factors in OS for malignant patients with pleural effusion. In addition, serum VEGF is also a prognostic biomarker associated with a poor prognosis in PFS for malignant patients with pleural effusion (HR 1.70; 95\% CI 1.00-2.89). The forest plots of each biomarker in serum are showed on Additional file 1: eFigure 17-25. Heterogeneity testing revealed heterogeneity $\left(I^{2}>50 \%\right)$ in WBC, NLR, albumin, LDH, and VEGF for PFS.

\section{Pleural effusion prognostic biomarkers for malignant patients}

Pleural effusion biomarkers are always the best material to test the pleural diseases. We also summarized the pleural effusion prognostic biomarkers for malignant patients. In these studies, 3 were about the prognostic value of neutrophils, 12 of $\mathrm{PH}, 10$ of total protein, 4 of albumin, 12 of glucose, 14 of LDH, 10 of VEGF and 5 of survivin.

The pooled data of forest plot is showed in Fig. 4. The results demonstrate that low $\mathrm{PH}$ (HR 1.95; 95\% CI 1.462.60), low glucose (HR 1.75; 95\% CI 1.18-2.61), high LDH (HR 1.47; 95\% CI 1.18-1.84), high VEGF (HR 1.99; 95\% CI 1.67-2.37) and high survivin (HR 2.90; 95\% CI 1.17-7.20) in pleural effusion are prognostic risk factors in OS for malignant patients. In addition, pleural effusion 


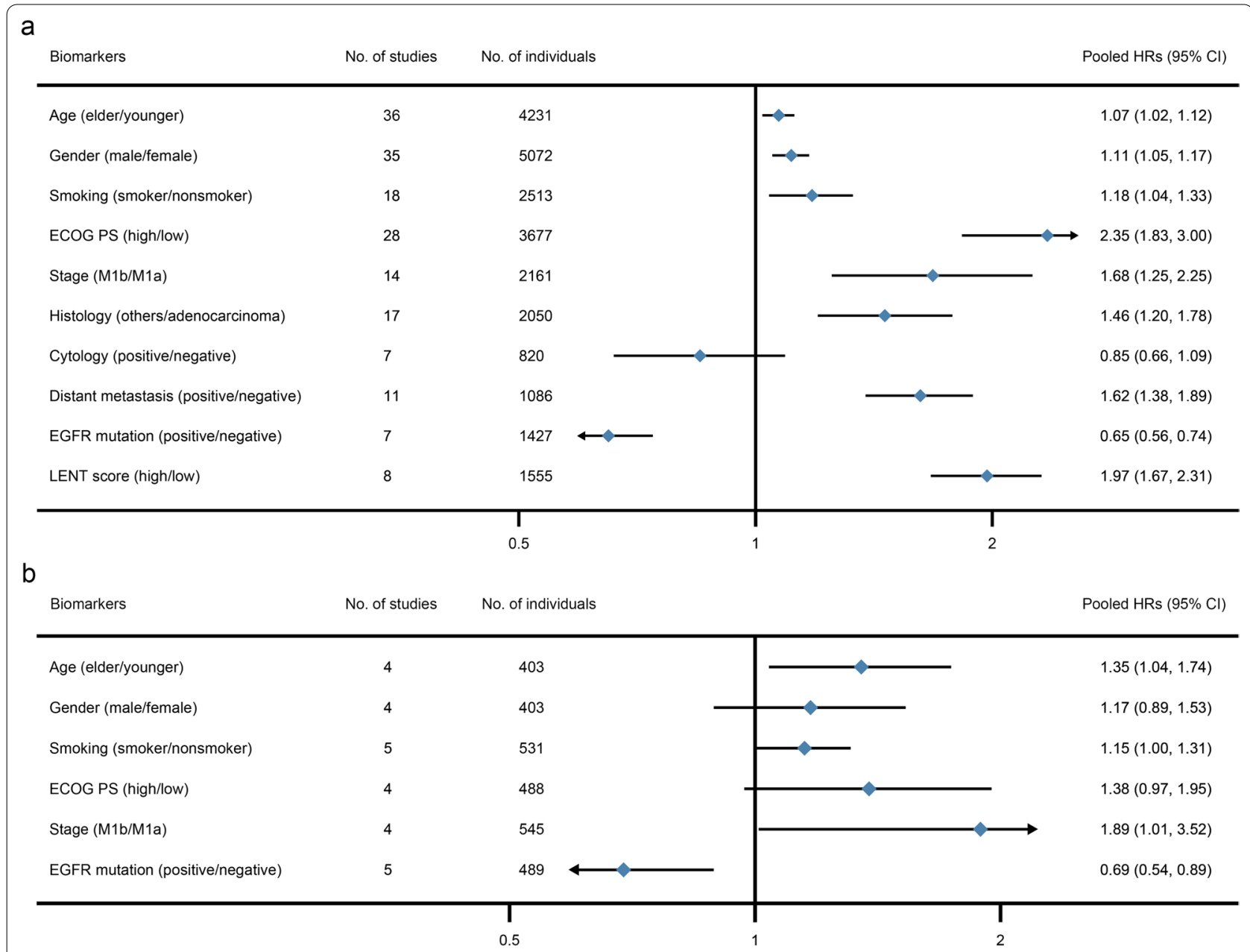

Fig. 2 Pooled HRs of the clinical parameters in prognostic value of OS (a) and PFS (b) in malignant patients with pleural effusion

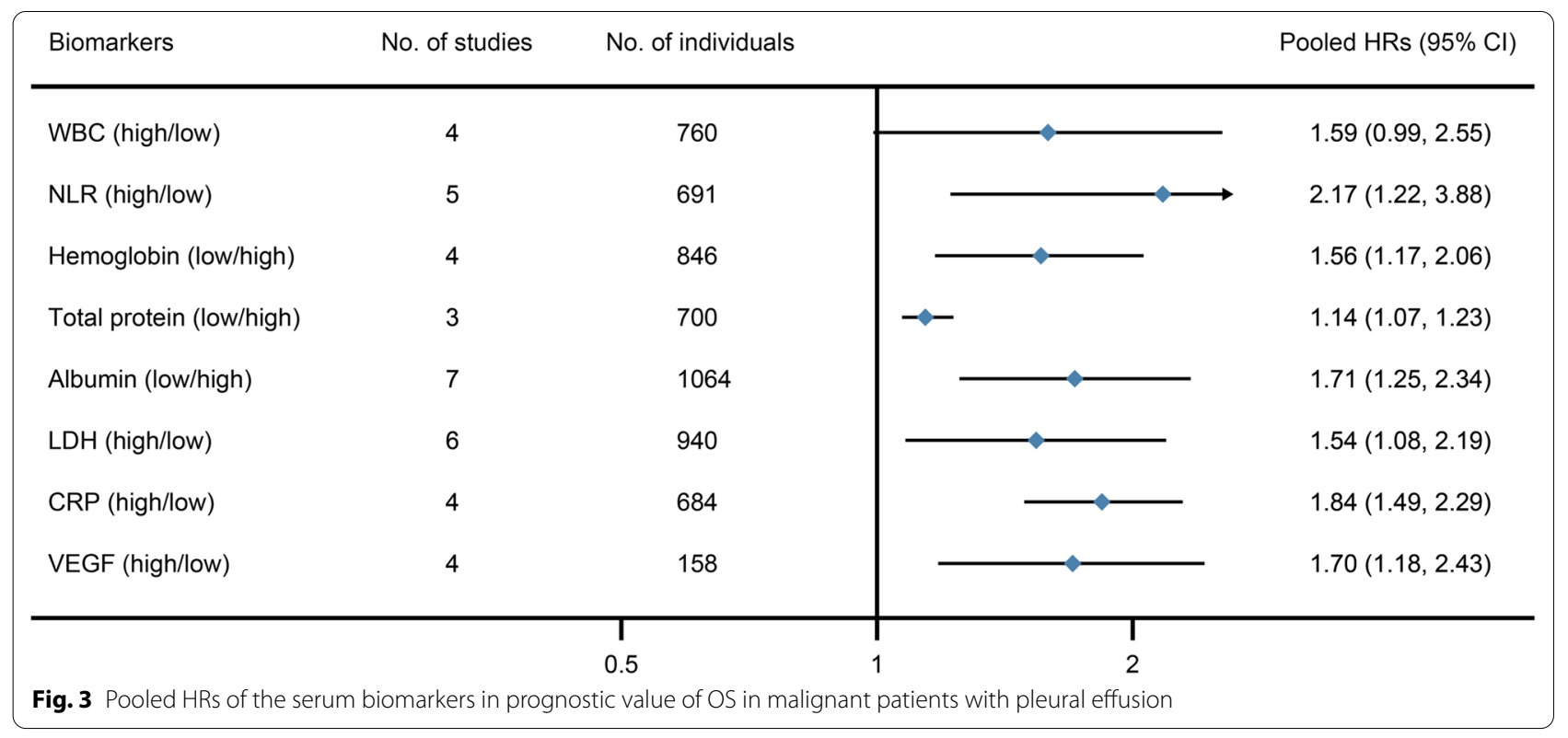




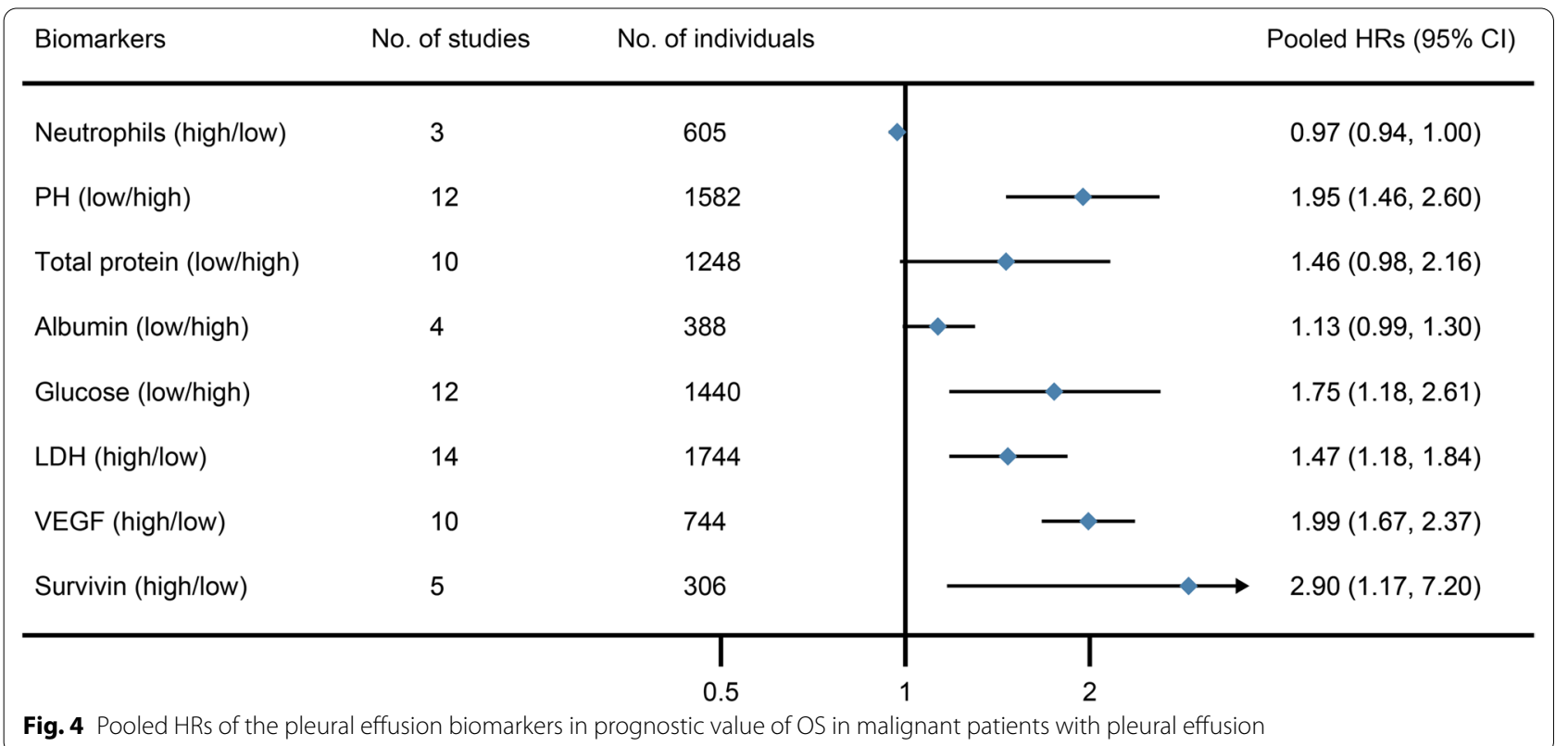

VEGF is also a prognostic biomarker associated with a poor prognosis in PFS for malignant patients with pleural effusion (HR 1.42; 95\% CI 1.02-2.00). The forest plots of each biomarker in serum are showed on Additional file 1: eFigure 26-34. Heterogeneity testing revealed heterogeneity $\left(I^{2}>50 \%\right)$ in $\mathrm{PH}$, total protein, glucose, LDH, VEGF for PFS and survivin.

\section{Subgroup analysis}

Significant heterogeneities were observed for the prognostic significance of age $\left(\mathrm{I}^{2}=58.9 \% ; \mathrm{P}<0.001\right)$, smoking status $\left(\mathrm{I}^{2}=64.7 \%\right.$; $\left.\mathrm{P}<0.001\right)$, ECOG PS $\left(\mathrm{I}^{2}=83.5 \%\right.$; $\mathrm{P}<0.001), \quad$ stage $\quad\left(\mathrm{I}^{2}=87.5 \% ; \quad \mathrm{P}<0.001\right), \quad$ histology $\left(\mathrm{I}^{2}=70.4 \% ; \mathrm{P}<0.001\right)$, serum WBC $\left(\mathrm{I}^{2}=87.7 \% ; \mathrm{P}<0.001\right)$, serum NLR $\left(\mathrm{I}^{2}=87.7 \% ; \mathrm{P}<0.001\right)$, serum albumin $\left(\mathrm{I}^{2}=61.2 \% ; \mathrm{P}<0.05\right)$, pleural effusion $\mathrm{PH} \quad\left(\mathrm{I}^{2}=88.5 \%\right.$; $\mathrm{P}<0.001)$, pleural effusion total protein $\left(\mathrm{I}^{2}=81.0 \%\right.$; $\mathrm{P}<0.001)$, pleural effusion glucose $\left(\mathrm{I}^{2}=86.2 \%\right.$; $\left.\mathrm{P}<0.001\right)$, pleural effusion LDH $\left(\mathrm{I}^{2}=70.5 \%\right.$; $\left.\mathrm{P}<0.001\right)$, pleural effusion VEGF $\left(\mathrm{I}^{2}=48.7 \% ; \mathrm{P}<0.05\right)$ and pleural effusion surviving $\left(\mathrm{I}^{2}=83.3 \%\right.$; $\left.\mathrm{P}<0.001\right)$ for overall survival in malignant patients with pleural effusion. For progressionfree survival, significant heterogeneities were observed for the prognostic significance of stage $\left(\mathrm{I}^{2}=87.8 \%\right.$; $\mathrm{P}<0.001)$, smoking status $\left(\mathrm{I}^{2}=64.7 \% ; \mathrm{P}<0.001\right)$, ECOG PS $\left(\mathrm{I}^{2}=83.5 \% ; \mathrm{P}<0.001\right)$, stage $\left(\mathrm{I}^{2}=87.5 \% ; \mathrm{P}<0.001\right)$, histology $\left(\mathrm{I}^{2}=70.4 \% ; \quad \mathrm{P}<0.001\right)$ and serum VEGF $\left(\mathrm{I}^{2}=80.7 \% ; \mathrm{P}=0.001\right)$ in malignant patients with pleural effusion. Thus, subgroup analyses were performed by categorizing subgroups by cancer types. As shown in Table 1, caner types in half of the studies were not specifically reported. In the subgroup analyses of the variables, there were no significant associations among cancer types (test for subgroup differences: $\mathrm{P}>0.05$ ) (Additional file 1: eFigure 35-41).

\section{Sensitivity analysis}

In order to find the source of heterogeneity, sensitivity analyses were performed in all prognostic biomarkers with $I^{2}>50 \%$ (Additional file 1: eFigure 42-59). Two studies (Burrows et al [15] and Özyurtkan et al [16]) significantly influence the pooled result of pleural effusion glucose for OS in malignant patients. We have excluded these two studies for the glucose pooled result.

\section{Publication bias}

We analyzed all biomarkers which include more than 10 studies. The contour-enhanced meta-analysis funnel plot of HR is presented in Additional file 1: eFigure 60-71. Publication bias was present in age, stage, histology, $\mathrm{PH}$ of pleural and LDH in pleural effusion with the Egger tests' $\mathrm{p}$ value $<0.05$.

\section{Discussion}

Pleural effusion is a common clinical problem in patients with cancer, and may be due to both primary thoracic tumor or to a metastatic spread in the chest and constitutes the first sign of disease in approximately $10 \%$ of patients [17]. This study systematically summarizes all possible prognostic factors of pleural effusion caused by malignancy and may give a hind about the treatment and prognosis of malignant patients. Our main findings 
indicate that except the common prognostic factors such as elder age, male gender, smoking status, more advanced disease and distant metastasis, many other indicators can be valuable prognostic factors for pleural effusion caused by malignancy. These indicators include the clinical parameters such as ECOG PS, non-adenocarcinoma histology, EGFR mutation and LENT score, the serum indicators such as NLR, hemoglobin, total protein, albumin, LDH, CRP and VEGF, and the pleural effusion indicators such as PH, glucose, LDH, VEGF and surviving.

A multi-marker strategy may be a much better approach in predicting MPE prognosis. LENT score is one of the most widely recognized scoring systems to predict survival in patients with malignant pleural effusion, calculated based on tumor type, ECOG PS, serum NLR, and pleural fluid LDH. Our study also confirms its effectiveness that LENT score shows an excellent prognostic value for malignant patients with pleural effusion, so do the LENT score calculation items (LDH, ECOG PS, NLR and tumor type). However, the LENT score has not included many important developments in the prognosis of pleural effusion [18]. In our study, positive EGFR mutation patients shows a better survival both in OS and PFS. As we know that lung adenocarcinoma with malignant pleural effusion is associated with a higher incidence of EGFR mutations [19], Abisheganaden et al. advice to modify the LENT score with EGFR mutation in lung adenocarcinoma patients [9]. The LENT score system was created as a robust prognostic score in order to aid in decision-making regarding treatment of the diverse populations of patients with malignant [7], so further modifications according to other prognostic factors may be needed to provide a better prognostic effect. The SELECT prognostication model, which included EGFR mutations, has recently been recognized as a more effective model for predicting survival in Asian MPE populations, and more studies are needed to evaluate the accuracy of this scoring system. Many biological parameters also show the prognostic value for MPE such as hemoglobin, albumin, CRP and VEGF in our study. PROMISE score combines these biological parameters and clinical parameters to accurately estimate 3 -month mortality [8]. This score includes pleural fluid tissue inhibitor of metalloproteinases as one of the evaluate indexes. Unfortunately, there is not enough study of this parameter for us to analyze.

Except the LENT score, modified LENT score, SELECT model and PROMISE score items, we also found that serum albumin, serum and pleural effusion VEGF, pleural effusion $\mathrm{PH}$, pleural effusion glucose and pleural effusion survivin are also valuable prognostic factors for malignant patients. VEGF is a potent angiogenic regulator with a crucial role in the initiation and progression of solid malignancies $[20,21]$ and MPE is associated with high levels of VEGF in serum and MPE [22, 23]. According to our analysis, the increased VEGF levels in pleural fluid and serum may associated with worse OS and PFS. In addition, pleural fluid analysis has not only diagnostic but also prognostic significance in patients with malignant effusion [24]. Our results have confirmed this view. These indictors provide a new direction for the prognosis of malignant patients with pleural effusion.

Neuron-specific enolase (NSE) [25], Cancer Antigen 153 (CA153) and Cancer Antigen 125 (CA125) [26] are validated for the diagnosis and prognosis of patients with cancer. However, there is still a lack of research on whether these biomarkers in pleural effusion have prognostic values on MPE patients. The prognosis value of other combined use of markers such as Cancer ratio [27] and Cancer ratio plus [28] in predicting survival of MPE should also be studied.

Our study has some limitations. First, the combinations of some factors have significant heterogeneity. The heterogeneity may come from the different types of cancer. Second, some of the funnel plots implied possible publication bias. We have already tried to include all studies that meet the criteria, but the publication bias cannot be avoided. Third, for the outcomes, only OS and PFS were involved. Although some studies demonstrate the disease-free survival, the number is too small to summarize.

\section{Conclusions}

Our findings suggest that for malignant patients with pleural effusion, LENT score and its items are valuable prognostic biomarkers, so do the clinical parameters like stage, distant metastasis, EGFR mutation, the serum biological parameters like hemoglobin, albumin, C-reaction protein, VEGF, and the pleural effusion biological parameters like PH, glucose, VEGF and survivin.

\section{Abbreviations}

MPE: Malignant pleural effusions; LDH: Lactate dehydrogenase; ECOG PS: Eastern Cooperative Oncology Group Performance Status; NLR: Neutrophil-tolymphocyte ratio; PRISMA: Preferred Reporting Items for Systematic Reviews and Meta-Analysis; OS: Overall survival; PFS: Progression-free survival; HR: Hazard ratio; NOS: Newcastle-Ottawa Scale; WBC: White blood cell counts; CRP: C-reactive protein; VEGF: Vascular endothelial growth factor.

\section{Supplementary Information}

The online version contains supplementary material available at https://doi. org/10.1186/s12935-022-02518-w.

Additional file 1. Original study protocol, supplementary tables and supplementary figures.

Acknowledgements Not applicable. 


\section{Authors' contributions}

SHZ designed the study and have been identified as the guarantor of the paper, taking responsibility for the integrity of the work as a whole, from inception to published article. PP, YY, DJ, and ZK contributed to the data collection. YY and DJ extracted the data, conducted the analysis, and produced the results figures and tables. PP and $Y Y$ wrote the initial draft of the manuscript. All coauthors read and commented on the manuscript.

\section{Funding}

This work was supported by grants from Beijing Municipal Administration of Hospitals' Mission Plan (No. SML20150301), Beijing Nova program (Z171100001117015), Beijing Talents Foundation (2017000021223ZK38) and "1351 Talents Program" of Beijing Chao-Yang Hospital (WXZXZ-2017-01 and CYXX-2017-35).

\section{Availability of data and materials}

Data sharing is not applicable to this article as no datasets were generated or analyzed during the current study.

\section{Declarations}

\section{Ethics approval and consent to participate} Not applicable.

\section{Consent for publication}

Not applicable.

\section{Competing interests}

All authors declare no conflict of interest.

\section{Author details}

${ }^{1}$ Department of Respiratory and Critical Care Medicine, Wuhan Pulmonary Hospital, Wuhan 430030, China. ${ }^{2}$ Department of Respiratory and Critical Care Medicine, Beijing Institute of Respiratory Medicine and Beijing Chao-Yang Hospital, Capital Medical University, 8 Gongti Nanlu, Chao-Yang District, Beijing 100020, China.

Received: 10 October 2021 Accepted: 8 February 2022 Published online: 24 February 2022

\section{References}

1. Feller-Kopman D, Light R. Pleural disease. N Engl J Med. 2018;378(8):740-51.

2. Taghizadeh N, Fortin M, Tremblay A. US hospitalizations for malignant pleural effusions: data from the 2012 national inpatient sample. Chest. 2017;151(4):845-54.

3. Asciak R, Rahman NM. Malignant pleural effusion: from diagnostics to therapeutics. Clin Chest Med. 2018;39(1):181-93.

4. Goldstraw P, Chansky K, Crowley J, et al. The IASLC lung cancer staging project: proposals for revision of the TNM stage groupings in the forthcoming (Eighth) edition of the TNM classification for lung cancer. J Thorac Oncol. 2016;11(1):39-51.

5. Han J, Cao S, Zhang K, et al. Fluorescence in situ hybridization as adjunct to cytology improves the diagnosis and directs estimation of prognosis of malignant pleural effusions. J Cardiothorac Surg. 2012;7(1):121.

6. Porcel JM, Gasol A, Bielsa S, Civit C, Light RW, Salud A. Clinical features and survival of lung cancer patients with pleural effusions. Respirology (Carlton, Vic). 2015;20(4):654-9.

7. Clive AO, Kahan BC, Hooper CE, et al. Predicting survival in malignant pleural effusion: development and validation of the LENT prognostic score. Thorax. 2014;69(12):1098-104

8. Psallidas I, Kanellakis NI, Gerry S, et al. Development and validation of response markers to predict survival and pleurodesis success in patients with malignant pleural effusion (PROMISE): a multicohort analysis. Lancet Oncol. 2018;19(7):930-9.

9. Abisheganaden J, Verma A, Dagaonkar RS, Light RW. An observational study evaluating the performance of LENT score in the selected population of malignant pleural effusion from lung adenocarcinoma in Singapore. Respiration. 2018;96(4):308-13.

10. Quek JC, Tan QL, Allen JC, Anantham D. Malignant pleural effusion survival prognostication in an Asian population. Respirology. 2020;25(12):1283-91.

11. Ryu JS, Ryu HJ, Lee SN, et al. Prognostic impact of minimal pleural effusion in non-small-cell lung cancer. J Clin Oncol. 2014;32(9):960-7.

12. Moher D, Liberati A, Tetzlaff J, Altman DG. Preferred reporting items for systematic reviews and meta-analyses: the PRISMA statement. BMJ (Clinical research ed). 2009;339:b2535.

13. Parmar MKB, Torri $V$, Stewart L. Extracting summary statistics to perform meta-analyses of the published literature for survival endpoints. Stat Med. 1998;17(24):2815-34.

14. Hartling L, Milne A, Hamm MP, et al. Testing the Newcastle Ottawa Scale showed low reliability between individual reviewers. J Clin Epidemiol. 2013;66(9):982-93.

15. Burrows $C M$, Mathews $C$, Colt HG. Predicting survival in patients with recurrent symptomatic malignant pleural effusions - an assessment of the prognostic values of physiologic, morphologic, and quality of life measures of extent of disease. Chest. 2000;117(1):73-8.

16. Ozyurtkan MO, Balci AE, Cakmak M. Predictors of mortality within three months in the patients with malignant pleural effusion. Eur J Intern Med. 2010;21(1):30-4.

17. Meriggi F. Malignant pleural effusion: still a long way to go. Rev Recent Clin Trials. 2019;14(1):24-30.

18. Rozman A, MokTSK. Is the LENT score already outdated? respiration. Int Rev Thorac Dis. 2018;96(4):303-4.

19. Wu SG, Gow CH, Yu CJ, et al. Frequent epidermal growth factor receptor gene mutations in malignant pleural effusion of lung adenocarcinoma. Eur Respir J. 2008;32(4):924-30.

20. Edelman MJ, Hodgson L Fau - Wang X, Wang X Fau - Christenson R, et al. Serum vascular endothelial growth factor and COX-2/5-LOX inhibition in advanced non-small cell lung cancer: Cancer and Leukemia Group B 150304. (1556-1380 (Electronic)).

21. Choi JH, Kim HC, Lim HY, et al. Vascular endothelial growth factor in the serum of patients with non-small cell lung cancer: correlation with platelet and leukocyte counts. Lung Cancer 2001;33(2-3):171-9.

22. Sack U, Hoffman M, Zhao XJ, et al. Vascular enclothelial growth factor in pleural effusions of different origin. Eur Respir J. 2005;25(4):600-4.

23. Yanagawa H, Takeuchi E, Suzuki Y, Ohmoto Y, Bando H, Sone S. Vascular endothelial growth factor in malignant pleural effusion associated with lung cancer. Cancer Immunol Immunother. 1999;48(7):396-400.

24. Bielsa S, Salud A, Martínez M, et al. Prognostic significance of pleural fluid data in patients with malignant effusion. Eur J Intern Med. 2008;19(5):334-9.

25. Zych J, Szturmowicz M, Sakowicz A, et al. Neuron-specific enolase (NSE) serum level as a prognostic factor in non-small cell lung cancer. Pneumonol Alergol Pol. 2002;70(5-6):278-83.

26. Ma YY, Wang H, Zhao WD, et al. Prognostic value of combined lactate dehydrogenase, C-reactive protein, cancer antigen 153 and cancer antigen 125 in metastatic breast cancer. Cancer Control. 2022;29:10732748211053150.

27. Verma A, Abisheganaden J, Light RW. Identifying malignant pleural effusion by a cancer ratio (Serum LDH: Pleural Fluid ADA Ratio). Lung 2016;194(1):147-53.

28. Verma A, Dagaonkar RS, Marshall D, Abisheganaden J, Light RW. Differentiating malignant from tubercular pleural effusion by cancer ratio plus (cancer ratio: pleural lymphocyte count). Can Respir J. 2016;2016:7348239.

\section{Publisher's Note}

Springer Nature remains neutral with regard to jurisdictional claims in published maps and institutional affiliations. 